\title{
Acides gras polyinsaturés trans : aspects métaboliques
}

\section{Polyunsaturated trans fatty acids: metabolic aspects}

Oléagineux, Corps Gras, Lipides. Volume 7, Numéro 1, 40-3, Janvier - Février 2000, Dossier : actes des Journées Chevreul "Corps gras, nutrition et santé, questions d'actualité" (Bordeaux, Pessac)

Auteur(s) : Jean-Louis SEBEDIO, Jean-Michel CHARDIGNY, INRA, Unité de nutrition lipidique, 17, rue Sully, BV 1540, 21034 Dijon, France.

Résumé : Les acides gras trans polyinsaturés sont formés à partir des acides gras indispensables (acides linoléique et a-linolénique) au cours des traitements technologiques comme par exemple le raffinage des huiles ou la friture [1, 2]. L'acide linolénique est plus sensible à l'isomérisation que l'acide linoléique. En effet, comme l'a montré Wolff dans une revue récente [3], le degré d'isomérisation de l'acide linolénique peut atteindre 30 à $35 \%$. On retrouve également ces pourcentages dans les formules infantiles et le lait de femme [1]. Les principaux isomères formés sont les composés monotrans, à savoir les 18:2-9trans,12cis, 18:2-9cis,12trans, 18:39 trans,12cis,15cis, et le 18:3-9cis,12cis,15-trans. Cependant, une température de désodorisation supérieure à $220^{\circ} \mathrm{C}$ entraîne la formation d'isomères di-trans [4]. À titre d'exemple, nous avons représenté dans le tableau le contenu de quelques huiles végétales en isomères des acides linoléique et a-linolénique. Compte tenu de leur présence dans I'alimentation, on peut s'interroger sur les conséquences nutritionnelles et biochimiques de leur ingestion.

Summary: Trans isomers of linoleic and linolenic acids are usually found after heat treatment of vegetable oils (refined and used frying oils). These trans fatty acids can be like their corresponding cis isomers be oxidized and therefore used for energy production. Some of them can also be converted into long chain fatty acids which are trans isomers of arachidonic, eicosapentaenoic and docosahexaenoic acids. However, their conversion is influenced by the position of the trans ethylenic bond. In general, a trans double bond is recognised as a saturated one by the acyltransferase, and a 9 cis double bond seem to be of importance for the esterification of the 18:3 isomers in human cholesteryl esters. 18:2 and 18:3 isomers having a trans ethylenic bond in the D 9 position are preferentially converted into trans dead end products (trans-20:2 and 20:3 isomers).

Keywords: trans fatty acid, oxidation, conversion. 


\section{ARTICLE}

\section{Conversion en métabolites supérieurs et incorporation dans les tissus}

Des travaux de Privett [5], de Beyers et Emken [6], Ratnayake [7] et Berdeaux [8] nous avaient montré que les isomères monotrans du 18:2 n-6 pouvaient être convertis en isomères trans de I'acide arachidonique. De même, Grandgirard et al. [9] et Chardigny et al. [10] ont montré que certains isomères du 18:3 n-3 pouvaient également être convertis chez le rat en isomères trans des acides éicosapentaénoïque $(20: 5 n-3)$ et docosahexaénoïque (22:6n-3). Ces isomères ont été retrouvés chez le rat dans tous les tissus. De plus, l'isomère 19t du 22:6 n-3 était retrouvé dans des structures nerveuses [2].

Ce n'est que très récemment que des données sur l'incorporation et la conversion de ces composés chez l'homme ont été obtenues [11]. En effet, une étude effectuée sur 88 volontaires sains, en Écosse, aux Pays-Bas et en France a révélé la présence d'isomères trans du 18:2 n-6 et du 18:3n-3 dans les différentes classes lipidiques du plasma à des taux comparables dans les trois pays, alors qu'il avait été montré qu'il existait un gradient de concentration du nord au sud pour les isomères trans en C18:1 [12]. Par ailleurs, on peut noter que les quantités les plus importantes de 18:2 et 18:3 trans se retrouvent dans les esters de cholestérol.

Il est important de constater que, parmi les isomères de l'acide linolénique que l'on peut trouver dans les huiles raffinées, seul l'isomère 18:3-9cis,12cis,15trans semble s'incorporer dans les esters de cholestérol plasmatiques alors que les deux isomères monotrans sont retrouvés dans les triacylglycérols et les phospholipides (figure 1). À cela, une explication possible : la lécithine cholestéryl acyltransférase (LCAT) est l'enzyme responsable de l'incorporation des acides gras aux esters de cholestérol plasmatiques et l'un de ses substrats préférentiels est le 18:2 n-6. L'isomère 18:3-9cis,12cis,15trans serait reconnu comme son homologue structural, ce qui ne serait pas le cas de l'autre isomère qui a une liaison trans en position 9, comme il a été décrit pour son incorporation dans les cardiolipides chez le rat [13].

Cette même étude (TRANSlinE) a également montré que l'apport d'isomères trans de l'acide alphalinolénique à raison de 0,6 \% de l'énergie pendant six semaines entraîne une augmentation de ceuxci dans les lipides plasmatiques. Par exemple, dans les phospholipides, les teneurs en 18:3 trans augmentent de 0,05 à $0,20 \%$ des acides gras totaux et un isomère géométrique de l'EPA ayant une liaison trans en position 17, donc métabolite du 18:3-9cis,12cis, 15trans est présent. En revanche, le produit de conversion du 18:3-9trans,12cis,15cis n'a pas été mis en évidence même après six semaines de régime [11].

\section{Importance de la position de la liaison trans pour la désaturation et l'élongation des acides gras trans polyinsaturés}

La conversion des isomères trans des acides linoléique et linolénique a récemment été étudiée par Brétillon et al. [14] en utilisant le modèle de foie de rat isolé et perfusé et des isomères d'acides gras trans de synthèse marqués au ${ }^{14} \mathrm{C}$. Une liaison trans entre les carbones 12 et 13 d'une molécule de 18:2 augmente entre 10 et 20 fois la désaturation en position 6 de cette molécule par rapport à une liaison de configuration cis $[6,14]$. Cette première étape est limitante dans la conversion de l'acide linoléique en acide arachidonique [15]. Mais ceci ne semble pas être applicable à l'isomère 18:2-9cis, 12 trans car, bien que ce dernier soit désaturé en position 6 en quantité beaucoup plus importante 
que l'acide linoléique, il est désaturé et allongé en isomère 20:4-5cis, 8cis, 11cis, 14trans comme l'est l'acide linoléique en acide arachidonique $[6,8]$. En revanche, si la double liaison la plus proche du méthyl terminal d'une molécule de 18:3 n-3 (la position 15) est de configuration trans, la désaturation en position 6 est diminuée de moitié par rapport à l'isomère cis [16] et la formation de 20:5-5cis, 8cis, 11cis, 14cis, 17trans l'est également [14]. Cette différence est encore plus marquée quand la double liaison est en position 9, puisque c'est d'un facteur 10 que la conversion en 20:5 est diminuée [14].

La voie classique de conversion des acides linoléique et alpha-linolénique en acides gras polyinsaturés à longue chaîne procède d'une série de désaturations et d'élongations. Cependant, les acides 18:2 n- 6 et 18:3n-3 peuvent par élongation directe donner des acides gras « produits de fin de chaîne » à savoir les 20:2 n-6 et 20:3 n-3.

Les isomères mono-trans des acides linoléique et alpha-linolénique sont également convertis en isomères trans de ces " produits de fin de chaîne ". Que ce soit sur une molécule de 18:2 n-6 ou de 18:3 n-3, la présence d'une double liaison trans en position 9 augmente la prise en charge par les élongases, car ce sont entre 2 à 5 fois plus de 20:2-11trans,14cis que de 20:2 n-6 qui sont formés respectivement à partir de l'isomère 18:2-9trans,12cis et de l'acide linoléique $[6,8,14]$, et 2,5 fois plus de 20:3-11trans, 14cis,17cis que de 20:3 n-3 qui sont formés à partir, respectivement, de I'isomère 18:3-9trans, 12cis,15cis et de l'acide alpha-linolénique [14]. II existe une controverse quant à la formation de l'acide 20:2-11cis,14trans, dont le précurseur est l'isomère 18:2-9cis,12trans. En effet, certains auteurs n'ont pas observé sa présence chez le rat in vivo $[7,8]$, alors que sa formation a été prouvée par d'autres $[6,14,17]$. La quantification des produits d'élongation des acides $\left[1-{ }^{14} \mathrm{C}\right]$ 18:2-9cis,12trans et linoléique a montré que, dans le foie, l'isomère 20:2-11cis,14trans est formé en quantité comparable à l'acide 20:2 n-6 [14]. En fait, l'élongation directe sans étape de désaturation représente l'essentiel du devenir des isomères qui sont les moins convertis par la voie classique de désaturation et d'élongation, c'est-à-dire les isomères 18:2-9trans, 12cis et 18:3-9trans,12cis,15cis. En effet, la formation de 20:2-11trans,14cis et de 20:3-11trans,14cis,17cis représente plus de $80 \%$ du total de la conversion de leurs précurseurs respectifs [14].

\section{Oxydation métabolique des isomères monotrans des acides linoléique et linolénique}

L'oxydation des acides linoléique et linolénique représente une voie importante de leur devenir métabolique [18-22]. Ainsi, Cunnane et Anderson [22] ont calculé la répartition des acides linoléique et alpha-linolénique alimentaires au terme d'une période de 26 jours en utilisant la mesure de la balance de l'acide gras comme décrite par Brétillon et al. [24]. L'estérification de ces acides gras dans les structures lipidiques complexes occupe la seconde place dans leur devenir, avec près de $19 \%$ pour l'acide linoléique et $11 \%$ pour l'acide alpha-linolénique. La part de chacun d'eux qui est utilisée à des fins de conversion en acides gras polyinsaturés à longue chaîne est relativement faible, 3 et 1,4 $\%$, respectivement. En outre, une part non négligeable des atomes de carbone issus de la betaoxydation des acides linoléique et alpha-linolénique entre dans la biosynthèse de novo d'acides gras non essentiels, tels les acides gras saturés ou mono-insaturés [25-30] ou le cholestérol [26-28]. Aussi, les valeurs d'oxydation des acides linoléique et alpha-linolénique de 76 et $85 \%$ respectivement publiées par Cunnane et Anderson apparaissent surestimées puisque la mesure de la balance des acides gras ne prend par en compte l'utilisation d'une partie du squelette carboné des acides linoléique et alpha-linolénique pour cette néosynthèse lipidique. 
Les premiers résultats de l'oxydation des acides gras trans ont été obtenus par Anderson et Coots [21] en utilisant un mélange équimoléculaire des deux isomères 18:2-trans uniformément marqués au ${ }^{14} \mathrm{C}$. Ils ont ainsi montré que l'acide linoléique est moins oxydé que ses isomères monotrans. Plus récemment, Brétillon et al. [23] ont montré que l'oxydation des isomères trans de l'acide linolénique déterminée par mesure directe $\mathrm{du}^{14} \mathrm{CO}_{2}$ présente un profil asymptotique avec un plateau atteint 12 heures après administration de l'acide gras radiomarqué (figure 2). Aucune différence n'est observée entre l'oxydation des deux isomères trans du 18:3 n-3 et l'acide linolénique au cours des 24 heures après administration des différents isomères. L'utilisation des isomères 18:3 trans est rapide puisque les maxima d'oxydation des isomères 15trans,9trans et du 18:2 n-6 sont observés $3 \mathrm{~h}, 4 \mathrm{~h}$ 30 et $3 \mathrm{~h}$ 30 respectivement après le gavage gastrique.

Pour ce qui est des isomères trans de l'acide linoléique (figure 3), il n'en est pas de même. En effet, I'isomère 18:2-9cis,12trans est plus oxydé que l'isomère 18:2-9trans,12cis et l'acide linoléique. Ce résultat permet de préciser les données publiées par Anderson et Coots [21]. Si l'on calcule la quantité de $\mathrm{CO}_{2}$ marqué produit par heure, on peut observer un pic d'oxydation du 18:2 n-6, $2 \mathrm{~h} 30$ après administration du produit marqué, alors que le pic d'oxydation des isomères 18:2-9cis,12trans et 18:2-9trans,12cis est atteint après $5 \mathrm{~h}$ et $4 \mathrm{~h} 30$ respectivement. Du fait de la multiplicité des phénomènes mis en jeu dans l'oxydation chez l'animal in vivo, il est difficile de déterminer l'origine de ce catabolisme facilité de l'isomère 18:2-9cis,12trans.

Des études chez l'humain, effectuées dans le cadre du projet TRANSlinE [31], nous ont montré également une différence d'oxydation du 18:2-9cis,12trans et pas de différence pour les isomères du 18:3.

\section{CONCLUSION}

Des études récentes ont montré que, chez l'homme comme chez l'animal, les isomères d'acides gras polyinsaturés sont incorporés dans les différentes classes lipidiques plasmatiques. Cependant, un seul isomère de l'acide linolénique, le 18:3-9cis,12cis,15trans est métabolisé en un isomère trans de l'acide éicosapentaénoïque. Celui-ci est incorporé dans les phospholipides plasmatiques et les plaquettes sanguines.

Les différents isomères du 18:2 n-6 et 18:3 n-3 sont oxydés de manière similaire chez l'homme et chez l'animal. II n'y a pas de différence d'oxydation des isomères du 18:3. En revanche, le 18:2$9 c i s, 12$ trans est plus oxydé que l'acide linoléique.

Des études chez l'animal ont également montré que le 18:2-9cis,12trans est un très bon substrat de la DELTA6 désaturase. En revanche, le 18:3DELTA9trans,12cis,15cis est dix fois moins converti en 20:5 n-3 que ne l'est l'acide linolénique. Les composés ayant une liaison trans en position 9 sont principalement convertis en " dérivés de fin de chaîne " (20:2 et 20:3) dont on ne connaît pas pour l'instant les effets biologiques éventuels.

\section{Remerciements}

Certaines études citées dans cette revue ont été réalisées grâce au financement de I'Union européenne par le biais du contrat FAIR 95-0594 et de la région Bourgogne (CERQUAVAL). 


\section{REFERENCES}

1. CHARDIGNY JM, SÉBÉDIO JL, BERDEAUX O (1996). Trans polyunsaturated fatty acids : occurrence and nutritional implications. Adv Appl Lipid Res, 2 : 1-33.

2. SÉBÉDIO JL, CHARDIGNY JM (1998). Biochemistry of trans polyunsaturated fatty acids. In : CHRISTIE WW, SÉBÉDIO JL, eds. Trans fatty acids in human nutrition. Dundee, Écosse : The Oily Press, $9: 191-215$.

3. WOLFF RL (1995). Ubiquité et caractéristiques des isomères trans de l'acide linolénique : une revue. $O C L, 2$ : 391-400.

4. HENON G, KEMENY Z, RECSEG K, ZWOBADA F, KOVARI K (1999). Deodorization of vegetable oils. Part I : modeling the geometrical isomerization of polyunsaturated fatty acids. J Am Oil Chem Soc, 76 : 73-81.

5. PRIVETT OS, STEARNS EMJ, NICKELL EC (1967). Metabolism of the geometric isomers of linoleic acid in the rat. J Nutr, $92: 303-10$.

6. BEYERS EC, EMKEN EA (1991). Metabolites of cis, trans, and trans,cis isomers of linoleic acid in mice and incorporation into tissue lipids. Biochim Biophys Acta, 1082 : 275-84.

7. RATNAYAKE WMN, CHEN ZY, PELLETIER G, WEBER D (1994). Occurrence of 5c,8c,11c,15teicosatetraenoic acid and other unusual polyunsaturated fatty acids in rats fed partially hydrogenated canola oil. Lipids, $29:$ 707-14.

8. BERDEAUX O, SÉBÉDIO JL, CHARDIGNY JM, et al. (1996). Effects of trans n-6 fatty acids on the fatty acid profile of tissues and liver microsomal desaturation in the rat. Grasas Aceit, 47 : 86-99.

9. GRANDGIRARD A, PICONNEAUX A, SÉBÉDIO JL, JULLIARD F (1998). Trans isomers of long chain n-3 polyunsaturated fatty acids in tissue lipid classes of rats fed with heated linseed oil. Reprod Nutr Dev, $38: 17-29$.

10. CHARDIGNY JM, SÉBÉDIO JL, GRANDGIRARD A, MARTINE L, BERDEAUX O, VATELE JM (1996). Identification of novel trans isomers of 20:5 n-3 in liver lipids of rat fed a heated oil. Lipids, $31: 165$ 8.

11. SÉBÉDIO JL, MENSINK RP, CHARDIGNY JM, BEAUFRÈRE B, VERMUNT S, ARMSTRONG RA, CHRISTIE WW, NIEMELA J, HENON G, RIEMERSMA RA (1999). Nutritional and health impact of trans polyunsaturated fatty acids in European populations, the TRANSLinE study. Design, method and baseline characteristics and adherence to dietary intervention. Eur J Chin Nutr, $53: 1-10$.

12. WOLFF RL (1994). Les isomères 18:1 trans dans I'alimentation des Européens. Évaluations quantitative et qualitative. $O C L, 1: 209-18$.

13. WOLFF RL, COMBE NA, ENTRESSANGLES B, SÉBÉDIO JL, GRANDGIRARD A (1993). Preferential incorporation of dietary cis-9,cis-12,trans-15 18:3 acid into rat cardiolipins. Biochim Biophys Acta, $1168: 285-91$. 
14. BRÉTILLON L, CHARDIGNY JM, NOËL JP, SÉBÉDIO JL (1998). Desaturation and chain elongation of $\left[1-{ }^{14} \mathrm{C}\right]$ mono-trans isomers of linoleic and alpha-linolenic acids in perfused rat liver. J Lipid Res, 39 : 2228-39.

15. MARCEL YL, CHRISTIANSEN K, HOLMAN RT (1968). The prefered metabolic pathway from linoleic acid to arachidonic acid in vitro. Biochim Biophys Acta, $164: 25-34$.

16. CHARDIGNY JM, BLOND JP, BRÉTILLON L, et al. (1997). Conversion of 18:3 DELTA9cis,12cis,15trans in rat liver microsomes. Lipids, 32 : 731-5.

17. BERDEAUX O, BLOND JP, BRÉTILLON L, et al. (1998). In vitro desaturation or elongation of monotrans isomers of linoleic acid by the rat liver microsomes. Mol Cell Biochem, 185 : 17-25.

18. LEYTON J, CRURY PJ, CRAWFORD MA (1987). Differential oxidation of saturated and unsaturated fatty acids in vivo in the rat. Br J Nutr, $57: 383-93$.

19. BRÉTILLON L, CHARDIGNY JM, SÉBÉDIO JL, POULLAIN D, NOË L JP, VATELE JM (1998). Oxidative metabolism of $\left[1-{ }^{14} \mathrm{C}\right]$ mono-trans isomers of linoleic and alpha-linolenic acids in the rat. Biochim Biophys Acta, 1390 : 207-14.

20. COOTS RH (1964). A comparison of the metabolism of cis, cis-linoleic,trans,trans-linoleic and a mixture of cis, trans and trans, cis-linoleic acids in the rat. J Lipid Res, 5 : 473-6.

21. ANDERSON RL, COOTS RH (1967). The catobolism of the geometric isomers of uniformly ${ }^{14} \mathrm{C}$ labeled DELTA9-octadecenoic acid and uniformly ${ }^{14} \mathrm{C}$-labeled DELTA9,12-octadecadienoic acid by the fasting rat. Biochim Biophys Acta, 144 : 525-31.

22. CUNNANE SC, ANDERSON MJ (1997). The majority of dietary linoleate in growing rats is betaoxidized or stored in visceral fat. J Nutr, $127: 146-52$.

23. BRÉTILLON L (1998). Approches du métabolisme des acides gras trans polyinsaturés chez l'homme et chez le rat. Doctorat de l'université Bordeaux I. Spécialité : Sciences des Aliments. $\mathrm{N}^{\circ} \mathrm{d}^{\prime}$ ordre : 1984.

24. BRÉTILLON L, SÉBÉDIO JL, CHARDIGNY JM (1999). Revue sur le devenir métabolique des acides gras trans polyinsaturés. $O C L, 6: 188-94$.

25. DHOPESWHARKAR GA, SUBRAMANIAN C (1975). Metabolism of linolenic acid in developing brain : I. Incorporation of radioactivity from $\left[1-{ }^{14} \mathrm{C}\right]$ linolenic acid into brain fatty acids. Lipids, $10: 238-41$.

26. SINCLAIR AJ (1975). Incorporation of radioactive polyunsaturated fatty acids into liver and brain of developing rat. Lipids, $10: 175-84$.

27. ANDERSON RL, CONNOR WE (1988). Uptake of fatty acids into liver and brain of developing rat. Lipids, $23: 286-90$.

28. CUNNANE SC, WILLIAMS SCR, BELL JD, et al. (1994). Utilization of uniformly labeled 13Cpolyunsaturated fatty acids in the synthesis of long-chain fatty acids and cholesterol accumulating in the neonatal rat brain. J Neurochem, 62 : 2429-36. 
29. SHEAFF RC, NATHANIELZ PW, BRENNA JT (1995). Interconversion of alpha-linolenate and docosahexaenoate in fetal Rhesus monkeys. FASEB J, 9 : A464.

30. SHEAFF GREINER RC, ZHANG Q, GOODMAN KJ, GIUSSANI DA, NATHANIELZ PW, BRENNA JT (1996). Linoleate, alpha-linolenate, and docosahexaenoate recycling into saturated and monounsaturated fatty acids is a major pathway in pregnant or lactating adults and fetal or infant rhesus monkeys. J Lipid Res, 37 : 2675-86.

31. BRÉTILLON L, CHARDIGNY JM, SÉBÉDIO JL, SCRIMGEOUR C, BERDAUX O, LOREAU O, GACHON P, BEAUFRÈRE B (1999). L'oxydation post-prandiale des acides gras polyinsaturés (AGPI) dépend de leur forme géométrique cis/trans chez le volontaire sain. Nutr Clin Métabol, 13 (Suppl. 1) : 51S.

Illustrations

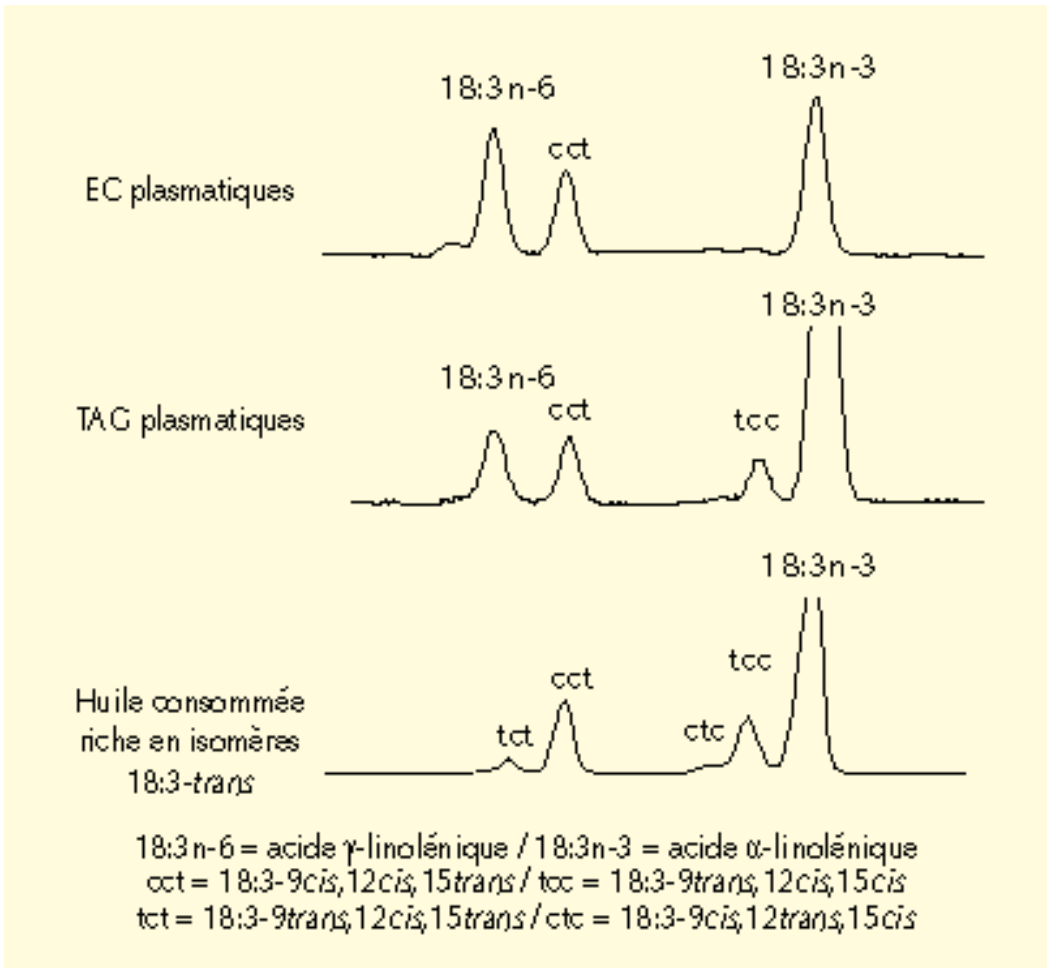

Figure 1. Chromatogrammes partiels des esters de cholestérol (EC) et triacylglycérols (TAG) plasmatiques d'un sujet humain astreint pendant 6 semaines à un régime riche en isomères trans de l'acide alpha-linolénique (0,6\% de l'apport calorique total) dont le profil en isomères 18:3 est tracé en bas de la figure (étude TRANSLinE [23]). 


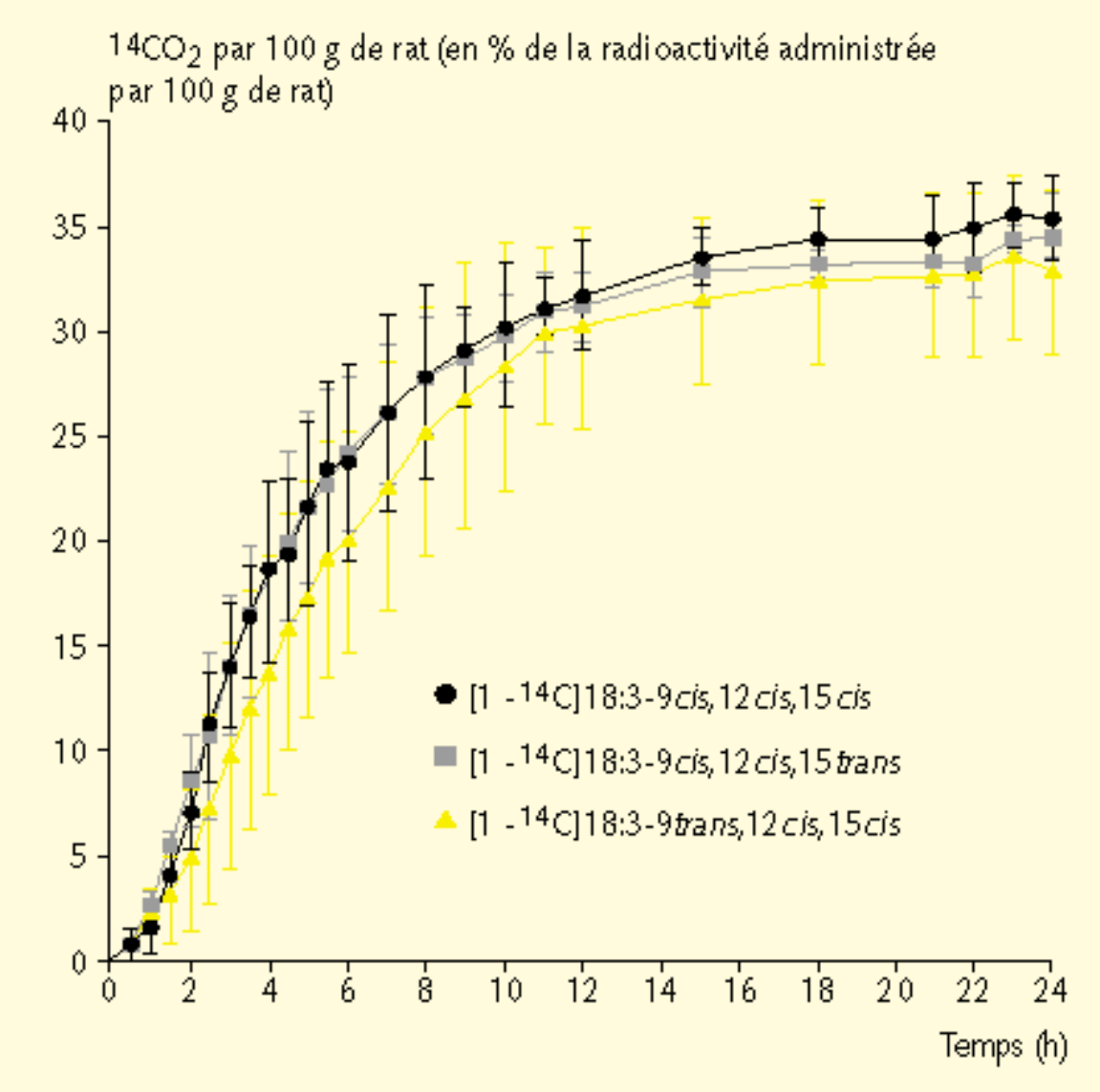

Figure 2. Oxydation métabolique de l'acide alpha-linolénique et de ses isomères monotrans (18:3-9cis,12cis,15trans et 18:3-9trans,12cis,15cis) radiomarqués $\left[1-{ }^{14} \mathrm{C}\right] \mathrm{chez}$ le rat adulte mâle soumis au jeûne [19] (reproduit avec la permission de Biochimica et Biophysica Acta). 


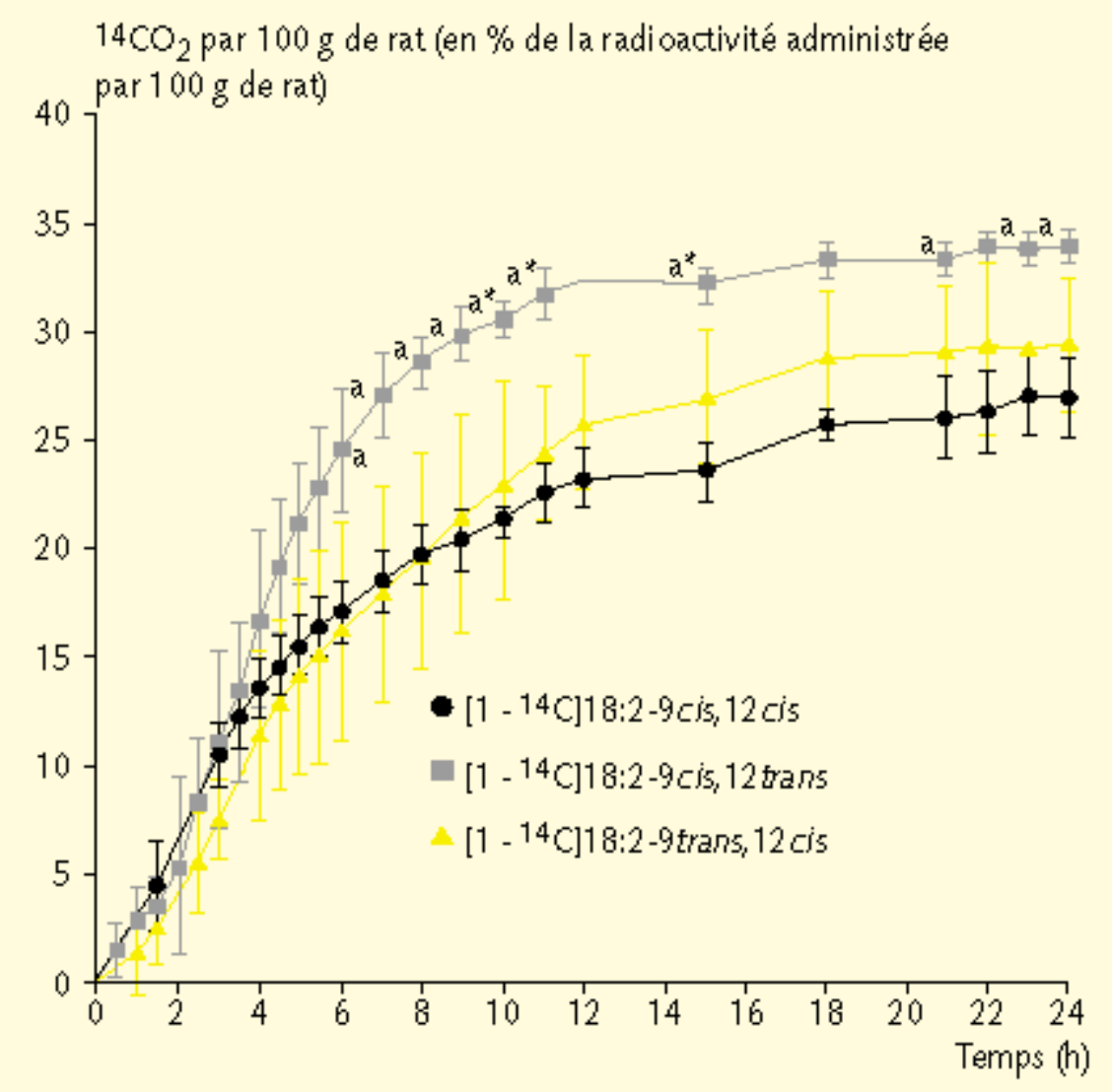

$a$ et $a^{*}$ : Significativement différents des valeurs correspondantes obtenues après administration des acides linoléqques et 18:2-9 trans, $12 \mathrm{cis}$

aे $p<0,05$ et 0,01 respectivement

Figure 3. Oxydation métabolique de l'acide linoléique et de ses isomères mono-trans radiomarqués $\left[1-{ }^{14} \mathrm{C}\right]$ chez le rat adulte mâle soumis au jeûne [19] (reproduit avec la permission de Biochimica et Biophysica Acta).

\begin{tabular}{|c|c|c|c|}
\hline Huile & Soja & Colza & Noix \\
\hline 18:2-9 ciş12trans & $0,05-0,51$ & $0,06-0,42$ & $0,140,27$ \\
\hline 18:2-9trans, 12 cis & $\leq 0,40$ & $0,01-0,35$ & $0,09-0,20$ \\
\hline 18:2-9 ciş12cis51,8-54,8 & $17,6-22,6$ & $58,6-59,9$ & \\
\hline 18:39 cis, 12cis, 15trans & $0,040,89$ & $0,341,46$ & $0,32-0,67$ \\
\hline 18:3-9trans, 12 cis, $15 c i s$ & $0,040,82$ & $0,27-1,31$ & $0,23-0,57$ \\
\hline 18:3-9 ciş12trans, 15 cis & $0,02-0,10$ & $0,040,28$ & $0,040,10$ \\
\hline 18:3-9trans, 12 cis, 15 trans & $\leq 0,27$ & $0,01-0,34$ & $0,02-0,04$ \\
\hline 18:3-9 ciş12ciş15cis & $5,3-7,1$ & $5,7-9,5$ & $11,6-11,8$ \\
\hline
\end{tabular}

Tableau. Teneurs (en \% des acides gras totaux) en isomères géométriques des acides linoléique et alpha-linolénique dans des huiles végétales raffinées (d'après [24]). 